\title{
High Dose Ofloxacin-induced Bimodal Hallucinations in a Four Years Old Child
}

\author{
Manish Pawar*, Shamsundar Jadhav* \\ Lifeline Clinic, Pune, India
}

\section{Dear Editor}

We read the case report article entitled "High Dose Ofloxacin-induced Bimodal Hallucinations in a 4 Years Old Child" by Bhattacharya et al." published in Clinical Psychopharmacology Neuroscience. We would like to inform you that, we are deeply concerned by some of the facts presented thereof. We would like to address some serious issues with the article here.

Authors have given a case report of a four year old girl child; who was admitted and treated with intravenous as well as oral ofloxacin as a first line treatment for acute gastroenteritis. We raise a concern about the use of ofloxacin as a first line antibiotic therapy in a pediatric case. Ofloxacin is contraindicated in children and growing adolescents due to possible effect of damage to growthplate cartilage as per the summary of product characteristics. ${ }^{2)}$

We disagree to the introductory statement of the article; 'Ofloxacin is a popular fluoroquinolone medication used in adults as well as children'. The use of fluoroquinolones in children is strictly restricted for the infections such as Mycobacterium tuberculosis, Pseudomonas aereginosa, Salmonella, Shigella and/or multi-drug resistant organisms. ${ }^{3)}$ Currently, fluoroquinolones (FQ) that are approved by the US Food and Drug Administration are being used in children for serious infections only. Ciprofloxacin is the only FQ approved by the European Medicines Agency for

Received: January 3, 2018/ Accepted: January 25, 2018

Address for correspondence: Shamsundar Jadhav, MBBS, MD Lifeline Clinic, Sunshine Hills, Undri Pisoli Road, Pune 411060 India

Tel: +91-9930210205

E-mail: drshyamjadhav@gmail.com

ORCID: https://orcid.org/0000-0002-7603-5213

*These authors contributed equally to this work. use serious pediatric conditions. Ciprofloxacin and levofloxacin are available as oral suspensions in many countries. An oral suspension of ciprofloxacin is available in the US, Canada, and 15 European countries, but systemic FQ use is not approved in Korea for any indication in children younger than 18 years. ${ }^{4}$

Another concern in the case report is use of an unstandardized and unsupervised oral dosage of ofloxacin in the child patient. The patient was prescribed oral ofloxacin $400 \mathrm{mg}, 1 / 4$ tablet twice daily and asked to follow-up after two days. Here, we want to point out to the negligence part on the prescribing physicians. The dosage used was 400 mg tablet; an adult dosage form and was prescribed as $1 / 4$ of a tablet. The concern here is, if the prescribing physician wanted to use dosage of $100 \mathrm{mg}$ twice daily, they should have chosen the $100 \mathrm{mg}$ tablet of ofloxacin. If the $100 \mathrm{mg}$ tablet was unavailable, then the administration should have been supervised. This would have minimized the possibility of medication error and the adverse effects due to it.

In the context of reported adverse effect of delirium in the given case where an unapproved drug was used, more careful treatment selection and therapeutic monitoring could have avoided the 'serious' side effect especially when safety profile of the drug have not been evaluated in the pediatric population.

\section{REFERENCES}

1. Bhattacharya A, Sharan R, Praharaj SK. High dose ofloxacin-induced bimodal hallucinations in a 4 years old child. Clin Psychopharmacol Neurosci 2017;15:416-417.

2. Generics UK T/A Mylan [Internet]. Hertfordshire: Generics [UK] Limited t/a Mylan; 2016 Mar [cited at 2017 Dec 18]. Available from: https://www.medicines.org.uk/emc/product/ 8480/smpc.

3. Goldman JA, Kearns GL. Fluoroquinolone use in paediatrics:

(a) This is an Open-Access article distributed under the terms of the Creative Commons Attribution Non-Commercial License (http://creativecommons.org/licenses/by-nc/4.0) which permits unrestricted non-commercial use, distribution, and reproduction in any medium, provided the original work is properly cited. 
focus on safety and place in therapy. In: The 18th WHO Expert Committee Meeting, Mar 21-25, 2011; Accra, Ghana. Section 6.2
4. Choi SH, Kim EY, Kim YJ. Systemic use of fluoroquinolone in children. Korean J Pediatr 2013;56:196-201. 